\title{
Change of temporal and support parameters of elderly gait by achilles tendon vibratory stimulation
}

\author{
Kiyoung Kwak ${ }^{1}$, Huigyun Kim², Dongwook Kim ${ }^{3}$ \\ ${ }^{1,2}$ Department of Healthcare Engineering, Graduate School, Chonbuk National University, \\ Jeonju, Republic of Korea \\ ${ }^{3}$ Department of Biomedical Engineering, College of Engineering, Chonbuk National University, \\ Jeonju, Republic of Korea \\ ${ }^{3}$ Research Center of Healthcare and Welfare Instrument for Aged, Chonbuk National University, \\ Jeonju, Republic of Korea \\ ${ }^{3}$ Corresponding author \\ E-mail: ${ }^{1} k y k w a k 86 @ n a t e . c o m,{ }^{2} h e k u n 1249 @ n a v e r . c o m,{ }^{3}$ biomed@jbnu.ac.kr
}

Received 22 May 2017; accepted 30 May 2017

DOI https://doi.org/10.21595/vp.2017.18655

Check for updates

Abstract. This study investigated the changes of temporal parameters and the support moments caused when local vibration stimulation was applied to the walking of the elderly. For this, $180 \mathrm{~Hz}$ vibration was applied to the Achilles tendon during walking. The intensity of applied vibration is two kinds of intensity of perceptual threshold and perceptual sub threshold. Three dimensional motion analysis was performed to investigate the temporal parameters and the support moments. When a vibration stimulus is applied, stride length, cadence and Gait velocity were decreased, and the support moments were increased from terminal stance. These results imply that local vibratory stimulation can lead to changes in the gait of the elderly.

Keywords: somatosensory, vibration, elderly, gait, perception threshold.

\section{Introduction}

Neurological and physiological changes occur with aging. As a neurological change, Delwaide [1] reported a decrease in motor units and sensitivity. In addition, increased reaction times, rate of brain loss and reduced levels of neurotransmitter production in the nervous system have been reported [2]. Physiological changes have reported loss of muscle strength, motor nerve, muscle fiber and loss of aerobic capacity [2-5]. These changes due to aging will cause changes in gait and will pose a potential risk. For this reason, many researches on the change of walking due to aging are progressing.

Winter et al. [6] reported a decrease in stride length and Gait velocity and an increase in double-support time in the elderly's walking compared to young adults. Judge et al. [7] reported a decrease in Gait velocity, step length, and single-support time, Similarly, Kerrigan et al. [8] reported a decrease in gait speed and step length. Riley et al. [9] reported a reduction in Gait speed and hip extension, while Devita et al. [10] reported fewer hip flexor moments, knee extensor moments, and plantar flexor moments. These studies can characterize the gait pattern of the elderly, and application studies using it are needed. The purpose of this study is to investigate the changes of vibration stimulation applied to the elderly's gait, based on the results of research on the elderly's gait and the effects of vibration on gait.

\section{Methods}

\subsection{Subjects}

A total of 9 elderly male participated in this study. This study was approved by Chonbuk National University Institutional Review Board (IRB File No. JBNU 2015-06-012). 


\subsection{Equipment}

A wired vibration stimulator that generates vibration was used to apply vibration stimulation on Achilles' tendon. And a function generator was used to adjust the frequency and intensity of vibration.

A total of 11 active infrared emitting markers were attached to major joints to measure the elderly gait.

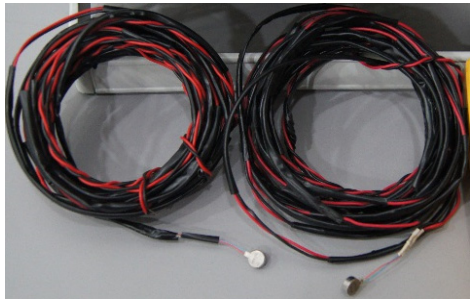

a)

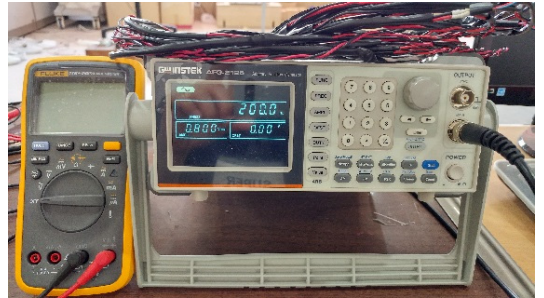

b)

Fig. 1. A wired vibrator and function generator

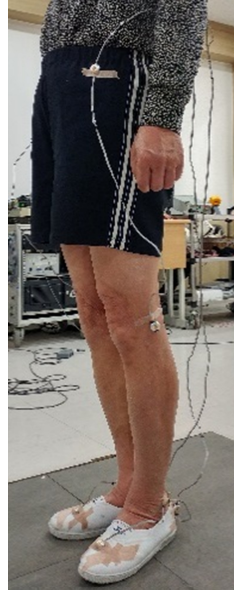

a)

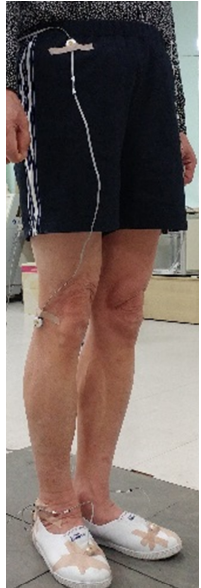

b)

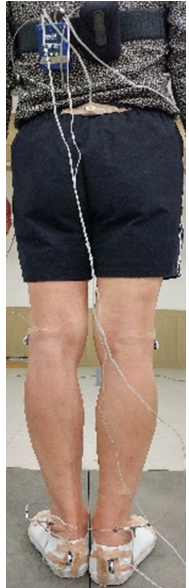

c)

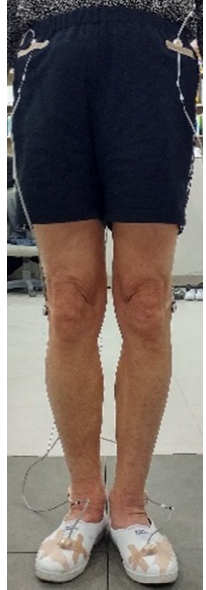

d)

Fig. 2. Infrared emitting makers attach locations

Total 3 position sensors (Optotrak Certus, Northern Digital Inc, Canada) and total 4 force plate (Bertec Co., Ltd, U.S.A) were used to collect infrared signals from the markers and ground reaction force during gait. SIMM software (MusculoGraphics Inc., USA) was used to derive temporal parameters and support moment.

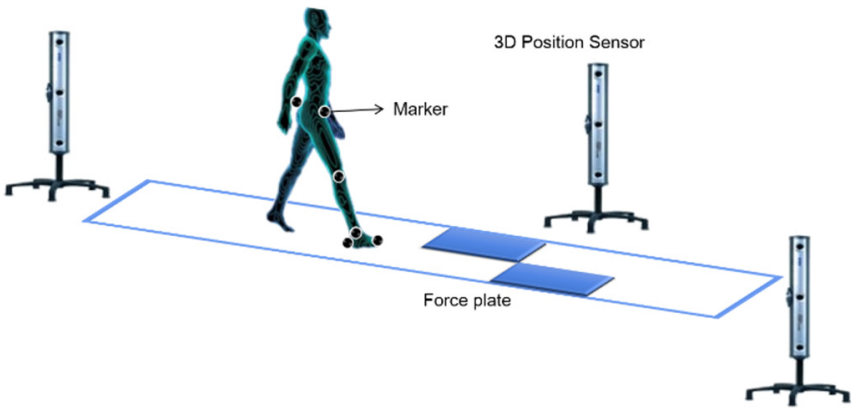

Fig. 3. Gait experiment 


\subsection{Protocol}

The subjects walked a walking trail at least $10 \mathrm{~m}$ in length at a pace of their choice. The $180 \mathrm{~Hz}$ vibration of perception threshold and perception sub-threshold intensity were randomly applied on Achilles' tendon during gait. The subjects performed gaits 3 times per each stimulation condition.

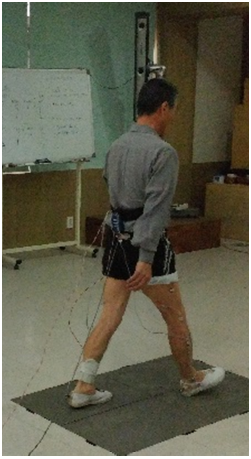

a)

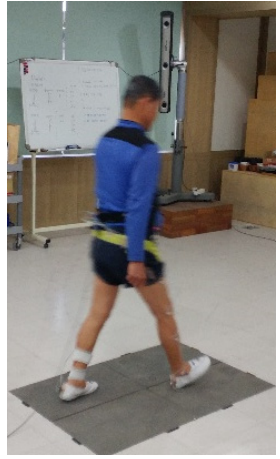

b)

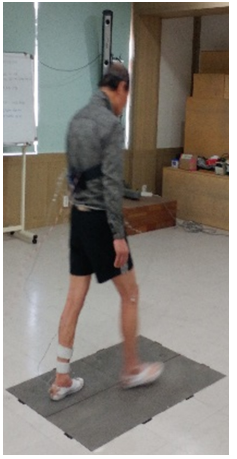

c)

Fig. 4. Subjects walking trial

\subsection{Analysis}

The stance phase (from heel contact to toe-off) was selected as the phase of analysis during gait. The temporal parameters and moment in ankle, knee, and hip joints were derived by using SIMM software. The support moment was calculated in accordance with the formula developed by Winter [6]. During the stance phase, the support moment was normalized as the body weight and then normalized as peak support moment.

\section{Results}

\subsection{Temporal parameters with vibration stimulation}

Temporal parameters of non-stimulation, Threshold stimulation and Sub-threshold stimulation conditions are presented in Table 1.

Table 1. Temporal parameters with vibratory stimulation on Achilles tendon

\begin{tabular}{|c|c|c|c|}
\hline ACT $180 \mathrm{~Hz}$ & Stride length $(\mathrm{cm})$ & Cadence $(\mathrm{steps} / \mathrm{min})$ & Gait velocity $(\mathrm{cm} / \mathrm{s})$ \\
\hline Non-stimulation & $123.61 \pm 4.15$ & $105.88 \pm 6.06$ & $108.73 \pm 3.63$ \\
\hline Threshold & $122.45 \pm 4.10$ & $102.35 \pm 2.55$ & $104.96 \pm 3.47$ \\
\hline Sub-threshold & $122.99 \pm 3.47$ & $105.14 \pm 3.37$ & $107.68 \pm 3.99$ \\
\hline
\end{tabular}

Gait velocity was slower in the threshold stimulation condition compared with the non-stimulation condition. Stride length of the threshold stimulation condition was shorter than that of the non-stimulation condition. Reduced cadence than that of the non-stimulation condition was presented in the threshold stimulation condition. Similarly, slower gait velocity, shorter stride length and reduced cadence were presented in the sub-threshold stimulation condition.

Winter et al. [6] reported that gait velocity, cadence and stride length were reduced and increased double-support stance time in the elderly. They interpreted that as gait adaptions of elderly related to less destabilizing gait. Thus, both vibration stimulation that further reduce temporal parameters will contribute positively to adaptation for the gait stabilization of the elderly. 


\subsection{Support moment patterns with vibration stimulation}

Support Moment curves of non-stimulation, Threshold stimulation and Sub-threshold stimulation conditions are illustrated in Fig. 5.

Support moment pattern of all conditions during stance phase are similar. However, after $50 \%$ of stance phase (start of terminal stance $\sim$ end of pre-swing), support moment of the threshold condition was increased compared with the non-stimulation condition. This increment also presented in the sub-threshold condition.

At this period, there are single-limb support then double-limb support. Simultaneously lower-limb extensor contributes to stabilization and forward progression of the body [11]. Thus, both vibration stimulation will contribute positively to stabilization and forward progression of the body of the elderly and further advancement swing phase.

The threshold vibratory stimulation affects extensor of lower-limb, thereby increasing the support moment between terminal stance and pre-swing, resulting in a decrease in temporal parameters. These results were also found in the sub-threshold vibratory stimulation. In other words, the vibratory stimulation contributes to the walking strategy of the elderly in order to further secure the stability of the walking, thereby making the walking more stable. On the other hand, it does not seem to make a significant contribution to forward progression. Thus, Future work should investigate the contribution of vibration stimulation to forward progression through power analysis of ankle joint during gait.

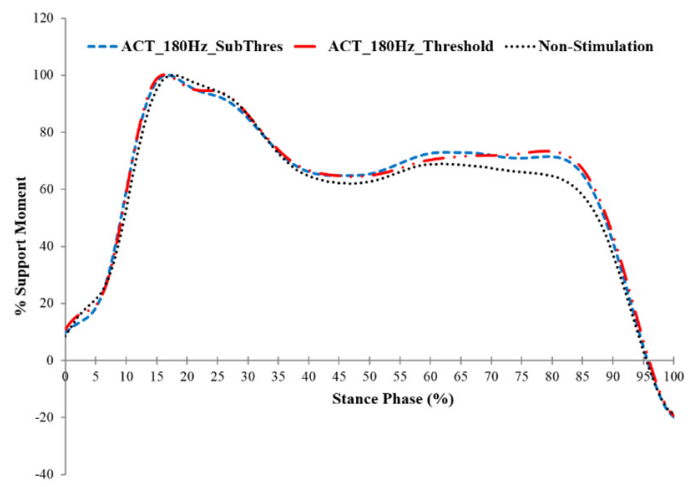

Fig. 5. Support moment with vibratory stimulation on Achilles tendon

\section{Conclusions}

This study examined that changes of temporal parameters and support moment with vibratory stimulation on Achilles' tendon during elderly gait. When threshold intensity vibration applied on Achilles' tendon, temporal parameters were reduced and support moment was increased in period of terminal stance and pre-swing. Through these results, it may suggest that vibratory stimulation on Achilles' tendon could affect positively to the gait stabilization and advancement of gait of elderly.

\section{Acknowledgements}

This study was supported by the National Research Foundation of Korea (NRF) Grant funded by the Korea Government (MSIP) (NRF-2014R1A2A1A11053073).

\section{References}

[1] Delwaide P. J. La marche du vieillard et ses problemes. Revue Medicale de Liege, Vol. 41, 1986, p. $864-867$. 
[2] Prince F., Corriveau H., Hebert R., Winter D. A. Gait in the elderly. Gait and Posture, Vol. 5, 1997, p. 128-135.

[3] Trueblood P. R., Rubenstein L. Z. Assessment of instability and gait in elderly persons. Comprehensive Therapy, Vol. 28, 1991, p. 20-29.

[4] Bendall M. J., Bassey E. J., Pearson M. B. Factors affecting walking speed of elderly people. Age and Ageing, Vol. 18, 1989, p. 327-332.

[5] Bassey E. J., Bendall, M. J., Pearson M. B. Muscle strength in the triceps surae and objectively measured customary walking activity in men and women over 65 years of age. Clinical Science, Vol. 1988, 1988, p. 85-89.

[6] Winter D. A., Patla A. E., Frank J. S., Walt S. E. Biomechanical walking pattern changes in the fit and healthy elderly. Physical Therapy, Vol. 70, 1990, p. 340-347.

[7] Judge J. O., Davis R. B., Ounpuu S. Step length reductions in advanced age: the role of ankle and hip kinetics. The Journals of Gerontology. Series A, Biological Sciences and Medical Sciences, Vol. 51, 1996, p. 303-312.

[8] Kerrigan D. C., Todd M. K., Croce U. D., Lipstiz L. A., Collins J. J. Biomechanical gait alterations independent of speed in the healthy elderly: evidence for specific limiting impairments. Archives of Physical Medicine and Rehabilitation, Vol. 79, 1998, p. 317-322.

[9] Riley P. O., Croce U. D., Kerrigan D. C. Effect of age on lower extremity joint moment contributions to gait speed. Gait and Posture, Vol. 25, 2001, p. 264-270.

[10] DeVita P., Hortobagyi T. Age causes a redistribution of joint torques and powers during gait. Journal of Applied Physiology, Vol. 88, 2000, p. 1804-1811.

[11] McGibbon C. A. Toward a better understanding og gait changes with age and disablement: neuromuscular adaptation. Exercise and Sport Sciences Reviews, Vol. 31, 2003, p. 102-108. 